\title{
On almost topological groups
}

\begin{abstract}
MADHU RAM*
Abstract. We introduce and study the almost topological groups which are fundamentally a generalization of topological groups. Almost topological groups are defined by using almost continuous mappings in the sense of Singal and Singal. We investigate some permanence properties of almost topological groups. It is proved that translation of a regularly open (resp. regularly closed) set in an almost topological group is regularly open (resp. regularly closed). And this fact gives us a lot of important and useful results of almost topological groups.
\end{abstract}

\section{INTRODUCTION}

A topological group is a group endowed with a topology which turns out the group operation and the inversion mapping (that is, $x \rightarrow x^{-1}$ ) continuous. Since the advent of this concept, it has been captured a great attention from different researchers and mathematicians. Many mathematicians have contributed significantly in the field of topological groups. Some first contributors to the theory of topological groups are A.D. Alexandroff, N. Bourbaki, M.I. Graev, S. Kakutani, E. van Kampen, A.N. Kolmogorov, A.A. Markov, Pontryagin, etc. Among those who contributed greatly to this field are A.V. Arhangel'skii, M.M. Choban, W.W. Comfort, D. Dikranjan, E. van Douwen, V.I. Malykhin, J. van Mill, B.A. Pasynkov, D. Shakhmatov, M. Tkachenko.

Recent literature of mathematics contains several similar notions and generalizations of topological groups. Semi-topological groups [10, 11], stopological groups [1, 4, 8], S-topological groups [1], quasi S-topological groups [6], irresolute topological groups [5, 9], quasi-irresolute topological groups [12] and paratopological groups [16] are well-known.

2010 Mathematics Subject Classification. Primary: 22A05, 54C08, 54 H99.

Key words and phrases. Regularly open sets, regularly closed sets, almost topological groups.

Full paper. Received 28 February 2018, revised 16 April 2019, accepted 17 April 2019, available online 27 April 2019.

*The author is supported by UGC-India under the scheme of NET-SRF fellowship. 
In this paper, we will study a new class of spaces, which we shall call almost topological groups. Basically, almost topological groups are a generalization of topological groups. We will present some examples of almost topological groups which are not topological groups. We also set forth some basic properties of almost topological groups. It is proved that translation of a regularly open (resp. regularly closed) set in an almost topological group is regularly open (resp. regularly closed), that any group homomorphism of almost topological groups which is R-continuous at the identity is almost continuous everywhere.

\section{Preliminaries}

Throughout the present paper, $(X, \tau)$ (or simply $X)$ means a topological space. For $A \subseteq X, C l(A)$ denotes the closure of $A$ and $\operatorname{Int}(A)$ denotes the interior of $A$. By $f: X \rightarrow Y$, we denote a map $f$ from a topological space $X$ to a topological space $Y$.

In 1963, N. Levine introduced the notion of semi-open sets in the literature of mathematics. He defined a set $A$ in a topological space $X$ to be semiopen [7] if there exists an open set $U$ in $X$ such that $U \subseteq A \subseteq C l(U)$; or equivalently, a subset $A \subseteq X$ is semi-open if and only if $A \subseteq C l(\operatorname{Int}(A))$. The complement of a semi-open set is semi-closed set; or equivalently, a subset $A$ of $X$ is semi-closed if $\operatorname{Int}(C l(A)) \subseteq A$. Every open set is semiopen but the converse is not true, in general. A subset $A$ of a topological space $X$ is called regularly open if $A=\operatorname{Int}(C l(A))$. The complement of a regularly open set is said to be regularly closed, or equivalently, a subset $A$ of $X$ is regularly closed if $A=C l(\operatorname{Int}(A))$. It is clear that every regularly open (closed) set is open (closed). However, the converse need not be true, in general. The finite intersection of regularly open sets is regularly open. Also, it is known that the closure of a semi-open set in $X$ is regularly closed and the interior of a semi-closed set in $X$ is regularly open.

A subset $A$ of a topological space $X$ is said to be $\delta$-open [17] if for each $x \in A$, there exists a regular open set $U$ in $X$ such that $x \in U \subseteq A$. The complement of a $\delta$-open set is called $\delta$-closed set [17]. The intersection of all $\delta$-closed sets in $X$ containing a subset $A \subseteq X$ is called the $\delta$-closure of $A$ and is denoted by $C l_{\delta}(A)$. It is known that a subset $A$ of $X$ is $\delta$-closed if and only if $A=C l_{\delta}(A)$. A point $x \in C l_{\delta}(A)$ if and only if $A \cap \operatorname{Int}(C l(U)) \neq \emptyset$ for each open set $U$ in $X$ containing $x$. The union of all $\delta$-open sets in $X$ that are contained in $A \subseteq X$ is called the $\delta$-interior of $A$ and is denoted by $\operatorname{Int}_{\delta}(A)$. The family of all regularly open (resp. regularly closed) sets of $X$ is denoted by $R O(X)$ (resp. $R C(X))$. If $A \in R O(X)$ and $B \in R O(Y)$, then $A \times B \in R O(X \times Y)$, where $X, Y$ are topological spaces.

In 1968, Singal and Singal [15] defined and investigated the notion of almost continuous mappings. They defined a function $f: X \rightarrow Y$ is said to be almost continuous if, for each $x \in X$ and for each open neighborhood $V$ 
of $f(x)$ in $Y$, there exists an open neighborhood $U$ of $x$ such that $f(U) \subseteq$ Int $(C l(V))$. In the same work, they proved a classical result saying that a function $f: X \rightarrow Y$ is almost continuous if and only if $f^{-1}(V)$ is open (resp. closed) in $X$, for every regular open (resp. regular closed) set $V$ in $Y$. Since then, many authors have accomplished an excellent work in the field of almost continuous mappings.

\section{Almost topological groups}

Before we start our main work, we introduce some notations. By $G$, we mean the group $(G, *)$ where '*' is a binary operation on $G$ under which $G$ is a group. For $x, y \in G$, we denote $x * y$ by $x y$ unless stated explicitly. We denote the inverse of an element $x$ in $G$ by $x^{-1}$ (i.e., under addition operation on $G, x^{-1}$ means $-x$ and under multiplication operation, $x^{-1}$ retains its notation and meaning). Translation of a set $A$ in a group $(G, *)$ (or simply, $G$ ) by an element $g$ is denoted by $g A$, defined by $g A=\{g * a: a \in A\}$. In this section, we introduce the notion of almost topological groups, present hosts of examples of almost topological groups and investigate several of their basic properties. Along with other results, we prove that translation of a regularly open (resp. regularly closed) set in an almost topological group is regularly open (resp. regularly closed).

Definition 3.1. Let $G$ be a group endowed with a topology $\tau$ such that

(1) the mapping $\phi: G \times G \rightarrow G$ defined by $\phi(x, y)=x y$, and

(2) the mapping $\psi: G \rightarrow G$ defined by $\psi(x)=x^{-1}$

are almost continuous, where $G \times G$ carries the product topology. Then the pair $(G, \tau)$ is called almost topological group. Equivalently, $(G, \tau)$ is an almost topological group if the following conditions are satisfied:

(1) For each $x, y \in G$ and each regularly open set $W$ in $G$ containing $x y$, there exist open neighborhoods $U$ and $V$ of $x$ and $y$, respectively, in $G$ such that $U * V \subseteq W$, and

(2) For each $x \in G$ and each regularly open set $V$ in $G$ containing $x^{-1}$, there exists an open neighborhood $U$ of $x$ in $G$ such that $U^{-1} \subseteq V$.

Remark 3.1. For any $A, B \subseteq G$, we denote $A * B$ by $A B$ and define as $A B=\{a b: a \in A, b \in B\}$ and $A^{-1}=\left\{a^{-1}: a \in A\right\}$. If $A=\{g\}$ for some $g \in G$, we denote $A * B$ by $g B$ and $B * A$ by $A g$.

Before we start discussing some general properties of almost topological groups. Let us present some examples of them.

Example 3.1. Consider the group $(G,+)$, where $G=\mathbb{R}$ be the set of real numbers, endowed with the standard topology $\mathcal{U}$. Then $(G, \mathcal{U})$ is an almost topological group.

Example 3.2. Let $(G, *)$ be any group with the discrete topology $\mathcal{D}$. Then $(G, \mathcal{D})$ is an almost topological group. 
In general, it is obvious from the definition that every topological group is an almost topological group, but the converse is not true in general. The following are the examples of almost topological groups which are not topological groups.

Example 3.3. Consider the group $G=\mathbb{Z}_{2}$ with the topology $\tau=\left\{\emptyset,\{0\}, \mathbb{Z}_{2}\right\}$. Then $(G, \tau)$ is an almost topological group which is not a topological group.

In fact, if $G$ is any group, $A$ proper subset of $G$ and $\tau=\{\emptyset, A, G\}$. Then $(G, \tau)$ is an almost topological group which is not a topological group.

Example 3.4. Let $G=\mathbb{R}$ be the additive group of real numbers and let $\tau$ be the topology on $G$ generated by the family $\{(a, b): a, b \in \mathbb{R}\} \cup\{(c, d) \cap D$ : $c, d \in \mathbb{R}\}$ where $D$ denotes the set of irrational numbers. Then $(G, \tau)$ is an almost topological group which is not a topological group.

Henceforth, we denote an almost topological group by $(G, \tau)$ (or simply $G$ when there is no chance of confusion). We turn now to some general properties of almost topological groups.

Theorem 3.1. Let $(G, \tau)$ be an almost topological group and let $g \in G$ be any element of $G$. Then:

(1) the mapping $h_{g}: G \rightarrow G$ defined by $h_{g}(x)=g x, \forall x \in G$, is almost continuous;

(2) the mapping $l_{g}: G \rightarrow G$ defined by $l_{g}(x)=x g, \forall x \in G$, is almost continuous.

Proof. (1) Let $x$ be any element of $G$ and let $W$ be a regular open set in $G$ containing $g x$. By definition 3.1, there exist open neighborhoods $U$ and $V$ of $g$ and $x$ respectively, in $G$ such that $U V \subseteq W$. In particular, $g V \subseteq W$ which means that $h_{g}(V) \subseteq W$. This indicates that $h_{g}$ is almost continuous at $x$ and hence $h_{g}$ is almost continuous.

(2) Pick up $x \in G$ and let $W \in R O(G)$ containing $x g$. Then there exist open sets $U$ in $G$ containing $x$ and $V$ in $G$ containing $g$ such that $U V \subseteq W$. This gives $U g \subseteq W$, i.e., $l_{g}(U) \subseteq W$. This shows that $l_{g}$ is almost continuous at $x$. Since $x$ was an arbitrary element of $G, l_{g}$ is almost continuous.

Theorem 3.2. Let $A$ be any regularly open set in an almost topological group $(G, \tau)$. The following are valid:

(1) $g A \in R O(G)$, for all $g \in G$.

(2) $A g \in R O(G)$, for all $g \in G$.

(3) $A^{-1} \in R O(G)$.

Proof. (1) Firstly, we show that $g A \in \tau$. Let $x \in g A$ be any element. Then by definition of almost topological groups, there exist open neighborhoods $U$ of $g^{-1}$ and $V$ of $x$ in $G$ such that $U V \subseteq A$. In particular, $g^{-1} V \subseteq A$. This is equivalent to the relation $V \subseteq g A$. This indicates that $x \in \operatorname{Int}(g A)$ and thus, 
$\operatorname{Int}(g A)=g A$. That is, $g A \in \tau$. Consequently, $g A \subseteq \operatorname{Int}(C l(g A))$. With an eye to the necessity of the problem, we have to show that $\operatorname{Int}(C l(g A)) \subseteq$ $g A$. Since $A$ is open, $C l(A) \in R C(G)$. By Theorem 3.1, $h_{g^{-1}}: G \rightarrow$ $G$ is almost continuous and as a result of this, it follows that $g C l(A)$ is closed. Therefore, $\operatorname{Int}(C l(g A)) \subseteq C l(g A) \subseteq g C l(A)$. This means that $g^{-1} \operatorname{Int}(C l(g A)) \subseteq C l(A)$. Since $\operatorname{Int}(C l(g A))$ is regularly open, it follows that $g^{-1} \operatorname{Int}(C l(g A)) \subseteq \operatorname{Int}(C l(A))=A$, i.e., $\operatorname{Int}(C l(g A)) \subseteq g A$. Thus $g A=\operatorname{Int}(C l(g A))$. Hence, job is done.

(2) The proof follows by similar arguments as in part (1) above.

(3) Let $y$ be any element of $A^{-1}$. Then there exists an open set $U$ in $G$ containing $y$ such that $U^{-1} \subseteq A \Rightarrow U \subseteq A^{-1}$. This proves that $y$ is an interior point of $A^{-1}$. Hence $A^{-1}$ is open. This results in $A^{-1} \subseteq$ $\operatorname{Int}\left(C l\left(A^{-1}\right)\right)$. It remains to prove $\operatorname{Int}\left(C l\left(A^{-1}\right)\right) \subseteq A^{-1}$. Since $A$ is open, $C l(A)$ is regularly closed and hence $C l(A)^{-1}$ is closed in $G$. Therefore, $\operatorname{Int}\left(C l\left(A^{-1}\right)\right) \subseteq C l\left(A^{-1}\right) \subseteq C l(A)^{-1} \Rightarrow \operatorname{Int}\left(C l\left(A^{-1}\right)\right) \subseteq \operatorname{Int}(C l(A))^{-1}=$ $A^{-1}$. Thus, $A^{-1}=\operatorname{Int}\left(C l\left(A^{-1}\right)\right)$, showing that $A^{-1} \in R O(G)$.

Corollary 3.1. Let $F$ be any regularly closed set in an almost topological group $G$. Then:

(1) $g F \in R C(G)$, for each $g \in G$.

(2) $F^{-1} \in R C(G)$.

Definition 3.2. A mapping $f: X \rightarrow Y$ is called almost open [15] if the image of any regularly open set is open, i.e., if for every $U \in R O(X), f(U)$ is open in $Y$.

Remark 3.2. Due to Theorem 3.2, it immediately follows that the mappings $h_{g}$ and $l_{g}$ in Theorem 3.1 are almost open.

Theorem 3.3. Let $A$ be any regularly open set in an almost topological group G. Then:

(1) $C l(g A)=g C l(A)$, for each $g \in G$.

(2) $C l(A g)=C l(A) g$, for each $g \in G$.

(3) $C l\left(A^{-1}\right)=C l(A)^{-1}$.

Proof. (1) Pick $x \in C l(g A)$ and consider $y=g^{-1} x$. Let $W$ be any open set in $G$ containing $y$. Then there exist open sets $U$ and $V$ in $G$ containing $g^{-1}$ and $x$ respectively, such that $U V \subseteq \operatorname{Int}(C l(W))$. By assumption, there is $a \in g A \cap V \Rightarrow g^{-1} a \in A \cap U V \subseteq A \cap \operatorname{Int}(C l(W)) \Rightarrow A \cap \operatorname{Int}(C l(W)) \neq$ $\emptyset \Rightarrow A \cap C l(W) \neq \emptyset$.

Since $A$ is open, $A \cap W \neq \emptyset$. That is, $x \in g C l(A)$. For the converse, let $y$ be any element of $g C l(A)$. Then $y=g x$ for some $x \in C l(A)$. In order to show that $g C l(A) \subseteq C l(g A)$, let $W$ be an open set in $G$ containing $g x$. Then there exist open sets $U$ in $G$ containing $g$ and $V$ in $G$ containing $x$ such that $U V \subseteq \operatorname{Int}(C l(W))$. Since $x \in C l(A), A \cap V \neq \emptyset$. There is $a \in A \cap V$. This gives $g a \in(g A) \cap \operatorname{Int}(C l(W)) \Rightarrow(g A) \cap C l(W) \neq \emptyset$. By Theorem 3.2, $g A$ 
is open and thus $(g A) \cap W \neq \emptyset$. Therefore, $y \in C l(g A)$. Combining the facts from above, we get $C l(g A)=g C l(A)$.

(2) Following up the same steps as in part (1) above, we can readily show $C l(A g)=C l(A) g$.

(3) Since $C l(A)$ is regularly closed, $C l(A)^{-1}$ is closed set in $G$. Therefore, the containment $A^{-1} \subseteq C l(A)^{-1}$ implies that $C l\left(A^{-1}\right) \subseteq C l(A)^{-1}$. Next, let $y \in C l(A)^{-1}$ be an arbitrary element. Then $y=x^{-1}$ for some $x \in C l(A)$. Let $V$ be any open set in $G$ containing $y$. Then there exists an open set $U$ in $G$ such that $x \in U$ with $U^{-1} \subseteq \operatorname{Int}(C l(V))$. Also, there is $a \in A \cap U$ which yields $a^{-1} \in A^{-1} \cap \operatorname{Int}(C l(V))$. That is, $A^{-1} \cap \operatorname{Int}(C l(V)) \neq \emptyset \Rightarrow$ $A^{-1} \cap C l(V) \neq \emptyset \Rightarrow A^{-1} \cap V \neq \emptyset$, since $A^{-1}$ is open. Therefore, $y \in C l\left(A^{-1}\right)$. Hence $C l\left(A^{-1}\right)=C l(A)^{-1}$.

Theorem 3.4. Let $A$ be any semi-open set in an almost topological group G. Then:

(1) $C l(g A) \subseteq g C l(A)$, for all $g \in G$.

(2) $C l(A g) \subseteq C l(A) g$, for all $g \in G$.

(3) $C l\left(A^{-1}\right) \subseteq C l(A)^{-1}$.

Proof. Here we give the proof of part (1) only. The proof for part (2) and (3) follow along similar lines.

(1) Since $A$ is semi-open, $C l(A)$ is regularly closed. By Theorem 3.1, $h_{g^{-1}}: G \rightarrow G$ is almost continuous. Therefore, $g C l(A)$ is closed. Hence $C l(g A) \subseteq g C l(A)$.

Theorem 3.5. Let $A$ be any regularly closed subset of an almost topological group $G$. The following assertions are valid:

(1) $\operatorname{Int}(g A)=g \operatorname{Int}(A)$, for all $g \in G$.

(2) $\operatorname{Int}(A g)=\operatorname{Int}(A) g$, for all $g \in G$.

(3) $\operatorname{Int}\left(A^{-1}\right)=\operatorname{Int}(A)^{-1}$.

Proof. (1) Since $A$ is regularly closed, $\operatorname{Int}(A)$ is regularly open in $G$. Consequently, $g \operatorname{Int}(A) \subseteq \operatorname{Int}(g A)$. Conversely, let $y \in \operatorname{Int}(g A)$ be arbitrary. Suppose that $y=g x$ for some $x \in A$. By hypothesis, it follows that $g A$ is closed and thereby $\operatorname{Int}(g A)$ is regularly open in $G$. Let $U$ and $V$ be open sets in $G$ containing $g$ and $x$ respectively, such that $U V \subseteq \operatorname{Int}(g A)$. Then $g V \subseteq g A$, whence it follows that $g V \subseteq g \operatorname{Int}(A)$. Thus, $\operatorname{Int}(g A) \subseteq g \operatorname{Int}(A)$. Hence the assertion follows.

Similarly, part (2) and (3) can be proved.

Theorem 3.6. Let $A$ be both semi-open and semi-closed subset of an almost topological group $G$. Then the following are valid:

(1) $C l(g A)=g C l(A)$, for each $g \in G$.

(2) $C l(A g)=C l(A) g$, for each $g \in G$.

(3) $C l\left(A^{-1}\right)=C l(A)^{-1}$. 
Proof. We only prove (1) and (3). The proof of part (2) follows along similar lines.

(1) Since $A$ is semi-open, $C l(A)$ is regularly closed, wherefrom it follows that $C l(g A) \subseteq g C l(A)$. Further, semi-openness of $A$ yields $C l(A)=$ $C l(\operatorname{Int}(A)) \Rightarrow g C l(A)=g C l(\operatorname{Int}(A))$. Since $A$ is semi-closed, $\operatorname{Int}(A)$ is regularly open in $G$. By Theorem 3.4, $g C l(A)=g C l(\operatorname{Int}(A))=C l(g \operatorname{Int}(A))$ $\subseteq C l(g A)$. From above calculations, we conclude that $C l(g A)=g C l(A)$.

(3) By hypothesis, it follows that $C l(A)$ is regularly closed and hence $C l(A)^{-1}$ is closed. Consequently, $C l\left(A^{-1}\right) \subseteq C l(A)^{-1}$. On other way round, since $A$ is semi-open, $C l(A)=C l(\operatorname{Int}(A)) \Rightarrow C l(A)^{-1}=C l(\operatorname{Int}(A))^{-1}$. Also, since $A$ is semi-closed, $\operatorname{Int}(A)$ is regularly open. By Theorem 3.3, $C l(A)^{-1}=C l\left(\operatorname{Int}(A)^{-1}\right) \subseteq C l\left(A^{-1}\right)$. This proves that $C l\left(A^{-1}\right)=C l(A)^{-1}$.

Using similar arguments, we obtain the following result:

Theorem 3.7. Under the same hypothesis of Theorem 3.6, the following are valid:

(1) $\operatorname{Int}(g A)=g \operatorname{Int}(A)$, for each $g \in G$.

(2) $\operatorname{Int}(A g)=\operatorname{Int}(A) g$, for each $g \in G$.

(3) $\operatorname{Int}\left(A^{-1}\right)=\operatorname{Int}(A)^{-1}$.

Proof. (1) Since $A$ is semi-closed, $\operatorname{Int}(A)$ is regularly open. By Theorem 3.1, $h_{g^{-1}}: G \rightarrow G$ is almost continuous. Therefore, $h_{g^{-1}}^{-1}(\operatorname{Int}(A))=g \operatorname{Int}(A)$ is open. Thus, $g \operatorname{Int}(A) \subseteq \operatorname{Int}(g A)$. Next, by hypothesis, it follows that $\operatorname{Int}(A)=\operatorname{Int}(C l(A)) \Rightarrow g \operatorname{Int}=g \operatorname{Int}(C l(A))$. Since $A$ is semi-open, $C l(A)$ is regularly closed. By Theorem 3.5, $g \operatorname{Int}(C l(A))=\operatorname{Int}(g C l(A)) \supseteq$ $\operatorname{Int}(g A)$. That is, $g \operatorname{Int}(A) \supseteq \operatorname{Int}(g A)$. Therefore, $\operatorname{Int}(g A)=g \operatorname{Int}(A)$.

Analogously, (2) and (3) can be proved.

Remark 3.3. By virtue of Examples 3.3 and 3.4, we can observe that both conditions 'semi-openness' and 'semi-closedness' on the set $A$ in Theorem 3.6 and Theorem 3.7 are essential.

Theorem 3.8. Let $A$ be any open set in an almost topological group $G$. Then $g A \subseteq \operatorname{Int}(g \operatorname{Int}(C l(A)))$ for each $g \in G$.

Proof. Since $A$ is open, $A \subseteq \operatorname{Int}(C l(A)) \Rightarrow g A \subseteq g \operatorname{Int}(C l(A))$. By Theorem $3.2, \operatorname{gInt}(C l(A))$ is open (in fact, regularly open). Hence $g A \subseteq$ $\operatorname{Int}(\operatorname{Int}(C l(A)))$.

In a similar style, we obtain the following result.

Theorem 3.9. Let $B$ be any closed subset of an almost topological group. Then $C l(g C l(\operatorname{Int}(B))) \subseteq g B$ for each $g \in G$.

Proof. Petty. 
Theorem 3.10. Let $G$ be an almost topological group. For any $A \subseteq G$, the following hold:

(1) $C l(g A) \subseteq g C l_{\delta}(A)$, for each $g \in G$.

(2) $g \operatorname{Int}_{\delta}(A) \subseteq \operatorname{Int}(g A)$, for each $g \in G$.

Proof. (1) Let $x$ be ny element of $C l(g A)$. Consider $y=g^{-1} x$ and let $W$ be any open neighborhood of $y$ in $G$. Then there exist open neighborhoods $U$ of $g^{-1}$ and $V$ of $x$ in $G$ such that $U V \subseteq \operatorname{Int}(C l(W))$.

Since $x \in C l(g A)$, there is $p \in(g A) \cap V$ and thus we always have, $g^{-1} p \in$ $A \cap(U V) \subseteq A \cap \operatorname{Int}(C l(W)) \Rightarrow A \cap \operatorname{Int}(C l(W)) \neq \emptyset$. Therefore, $y \in C l_{\delta}(A)$; that is, $x \in g C l_{\delta}(A)$.

(2) Let $y \in g \operatorname{Int}_{\delta}(A)$. Then $y=g x$ for some $x \in \operatorname{Int}_{\delta}(A)$. This means that there exists a regular open set $U$ in $G$ such that $x \in U \subseteq A$. In a sense, $\operatorname{Int}(C l(U)) \subseteq A \Rightarrow g \operatorname{Int}(C l(U)) \subseteq g A$. This gives $y=g x \in g \operatorname{Int}(C l(U)) \subseteq$ $g A$. By Theorem 3.2, $g \operatorname{Int}(C l(U))$ is open and thus, $y \in \operatorname{Int}(g A)$. Hence $g \operatorname{Int}_{\delta}(A) \subseteq \operatorname{Int}(g A)$.

Definition 3.3. A mapping $f: X \rightarrow Y$ is called R-continuous [2,3] if for each $x \in X$ and each regularly open set $V$ in $Y$ containing $f(x)$, there exists a regularly open set $U$ in $X$ containing $x$ such that $f(U) \subseteq V$.

Theorem 3.11. Let $G$ and $H$ be almost topological groups and $f: G \rightarrow H$ be a group homomorphism. If $f$ is $R$-continuous at $e_{G}$, then $f$ is almost continuous everywhere.

Proof. Let $x$ be any element of $G$. Let $V$ be a regularly open set in $H$ containing $y=f(x)$. By Theorem $3.2, y^{-1} V$ is regularly open set in $H$ containing $e_{H}$. By hypothesis, there exists a regularly open set $U$ in $G$ containing $e_{G}$ such that $f(U) \subseteq y^{-1} V$. This implies that $f(x U) \subseteq V$. By above observations, $x U$ is open, and thereby it follows that $f$ is almost continuous at $x$. Hence $f$ is almost continuous everywhere.

Theorem 3.12. Let $A$ be an open set in an almost topological group $G$. Then $C l_{\delta}(g A)=g C l_{\delta}(A)$ for each $g \in G$.

Proof. Since $C l_{\delta}(A)$ is regularly closed, by Corollary 3.2.1, $g C l_{\delta}(A)$ is regularly closed. Consequently, $C l_{\delta}(g A) \subseteq g C l_{\delta}(A)$. For the reverse inclusion, let $y \in g C l_{\delta}(A)$. Then $y=g x$ for some $x \in C l_{\delta}(A)$. For any open neighborhood $W$ of $y$, we obtain open neighborhoods $U$ of $g$ and $V$ of $x$ in $G$ satisfying $U V \subseteq \operatorname{Int}(C l(W))$. Our aim is to show $(g A) \cap \operatorname{Int}(C l(W)) \neq \emptyset$. Now, since $x \in C l_{\delta}(A), A \cap \operatorname{Int}(C l(V)) \neq \emptyset \Rightarrow A \cap C l(V) \neq \emptyset$. By hypothesis, $A \cap V \neq \emptyset$. Let $h \in A \cap V$. From this, we have $g h \in(g A) \cap(U V) \subseteq$ $(g A) \cap \operatorname{Int}(C l(W)) \Rightarrow(g A) \cap \operatorname{Int}(C l(W)) \neq \emptyset$. Hence $y \in C l_{\delta}(g A)$. This proves $g C l_{\delta}(A) \subseteq C l_{\delta}(g A)$. In conclusion, $C l_{\delta}(g A)=g C l_{\delta}(A)$.

A regularly open subset $U$ of an almost topological group $G$ containing $x \in G$ is called regularly open neighborhood of $x$. The collection of all regularly open neighborhoods of $x \in G$ is denoted by $\mathcal{N}_{x}$. 
Theorem 3.13. Let $G$ be an almost topological group. Then for every $V \in$ $\mathcal{N}_{e_{G}}$, there exists an open neighborhood $U$ of $e_{G}$ such that $U U \subseteq V$.

Proof. Since $e_{G} * e_{G}=e_{G}$, there exist open neighborhoods $A$ and $B$ of $e_{G}$ such that $A B \subseteq V$. Let $U=A \cap B$. Then $U$ is open neighborhood of $e_{G}$ such that $U U \subseteq V$. It completes the proof.

Theorem 3.14. Let $G$ be an almost topological group. Then for every $V \in$ $\mathcal{N}_{x}$, there exists an open neighborhood $U$ of $e_{G}$ such that $x U U \subseteq V$.

Proof. By Theorem 3.2, $x^{-1} V$ is regularly open neighborhood of $e_{G}$. By Theorem 3.13, there exists an open neighborhood $U$ of $e_{G}$ such that $U U \subseteq$ $x^{-1} V \Rightarrow x U U \subseteq V$.

Theorem 3.15. Let $G$ be an almost topological group. Then for every $U \in$ $\mathcal{N}_{e_{G}}, C l(U) \subseteq U^{-1} U$.

Proof. Let $x \in C l(U)$. Since $x U$ is open neighborhood of $x, U \cap(x U) \neq \emptyset$. Therefore, we obtain an equation, $g=x h$ for some $g, h \in U$. This gives $x=h^{-1} g \in U^{-1} U$. Hence the assertion follows.

Definition 3.4. A topological space $X$ is called almost regular [13] if for each regularly closed set $F$ in $X$ and each $x \notin F$, there exist disjoint open sets $U$ and $V$ in $X$ such that $x \in U$ and $F \subseteq V$.

Conjecture. Every almost topological group is almost regular.

Definition 3.5. A topological space $X$ is called nearly compact [14] if every open cover of $X$ has a finite subcover the interiors of the closures whose members cover $X$.

Theorem 3.16. If $A$ and $B$ are compact subsets of an almost topological group $G$, then $A B$ is nearly compact.

Proof. Let $A$ and $B$ be compact subsets of $G$. Since the image of a compact set under almost continuous mappings is nearly compact, by definition of an almost topological group, it follows that $A B$ is nearly compact.

\section{Acknowledgement}

I would like to thank the anonymous reviewers for their valuable comments/suggestions regarding the improvement of this manuscript. I must thank the editor for his kind cooperation throughout process of this manuscript.

\section{REFERENCES}

[1] M. S. Bosan, M. D. Khan, L. D. R. Kocinac, On s-Topological Groups, Math. Moravica, 18 (2) (2014), 35-44.

[2] D. A. Carnahan, Some properties related to compactness in topological spaces, Ph.D thesis, University of Arkansas, 1973. 
[3] Z. Duszynski, Almost continuity, regular set-connected mappings and some separation axioms, 39 (4) (2006), 939-948.

[4] M. D. Khan, M. S. Bosan, A note on s-topological groups, Life Sci J., 11 (7s) (2014), $370-374$.

[5] M. D. Khan, A. Siab, L. D. R. Kocinac, Irresolute Topological Groups, Math. Moravica, 19 (1) (2015), 73-80.

[6] M. D. Khan, S. Habib, M. S. Bosan, Quasi S-Topological Groups, Life Sci J., 27 (1) (2015), 53-57.

[7] N. Levine, Semi-open sets and semi-continuity in topological spaces, Amer. Math. Monthly, 70 (1963), 36-41.

[8] R. Noreen, M. D. Khan, Semi-connectedness in s-topological groups, J. Adv. Stud. Topol., 7 (2) (2016), 54-59.

[9] R. Noreen, M. S. Bosan, M. D. Khan, Semi-connectedness in Irresolute Topological Groups, Life Sci J., 27 (6) (2015), 4981-1985.

[10] T. Oner, M. B. Kandemir, B. Tanay, Semi-Topological Groups with Respect to Semicontinuity and irresoluteness, J. Adv. Stud. Topol., 4 (3) (2013), 23-28.

[11] T. Oner, A. Ozek, On Semi Topological Groups With Respect to Irresoluteness, Int. J. Recent Sci Res, 6 (12) (2015), 7914-7916.

[12] T. Oner, A. Ozek, A note on quasi-irresolute topological groups, J. Linear Topol. Algebra, 5 (1) (2016), 41-46.

[13] M. K. Singal, S. P. Arya, On almost regular spaces, Glasnik Mat. 4 (24) (1969), 89-99.

[14] M. K. Singal, A. Mathur, On nearly compact spaces, Boll. U. M. I., 4 (1969), 702-710.

[15] M. K. Singal, A. Rani, On almost continuous mappings, Yokohama Math. J., 16 (1968), 63-73.

[16] M. Tkachenko, Paratopological and semitopological groups vs topological groups, In: K.P. Hart, J. van Mill, P. Simon (eds.), Recent Progress in General Topology III, Atlantis Press., 2014, 825-882.

[17] N. V. Velicko, H-closed topological spaces, Amer. Math. Soc. Transl., 78 (2) (1968), $103-118$.

\author{
MADHU RAM \\ DEPARTMENT OF MATHEMATICS \\ UNIVERSITY OF JAMMU \\ JK-180006 \\ INDIA \\ E-mail address: madhuram0502@gmail.com
}

